\title{
Management of non-small cell lung cancer with resistance to epidermal growth factor receptor tyrosine kinase inhibitor: case discussion
}

\author{
Yuyan Zheng ${ }^{1}$, Min Zhou ${ }^{2}$, Surein Arulananda ${ }^{3}$, Sang-Won Um ${ }^{4}$, Hecheng $\mathrm{Li}^{1}$ \\ ${ }^{1}$ Department of Thoracic Surgery, ${ }^{2}$ Department of Respiratory Medicine, Ruijin Hospital, Shanghai Jiao Tong University School of Medicine, \\ Shanghai 200025, China; ${ }^{3}$ Department of Medical Oncology, Olivia Newton-John Cancer and Wellness Centre, Heidelberg, Australia; ${ }^{4}$ Division of \\ Pulmonary and Critical Care Medicine, Department of Medicine, Samsung Medical Center, Sungkyunkwan University School of Medicine, Seoul, \\ South Korea \\ Correspondence to: Hecheng Li, MD, PhD. Department of Thoracic Surgery, Ruijin Hospital, Shanghai Jiao Tong University School of Medicine, 197 \\ Ruijin 2nd Road, Shanghai 200025, China. Email: lihecheng2000@hotmail.com.
}

Submitted Jan 14, 2020. Accepted for publication Feb 02, 2020.

doi: $10.21037 /$ jtd.2020.01.65

View this article at: http://dx.doi.org/10.21037/jtd.2020.01.65

\section{Introduction}

First and second-generation epidermal growth factor receptor (EGFR) tyrosine kinase inhibitors (TKIs) have been widely employed as first line therapy for advanced non-small cell lung cancer (NSCLC) harboring EGFR mutations (1-3). However, most patients experience disease progression within 2 years due to acquired resistance. Subsequent therapeutic strategies should be carefully decided for each individual depending on mechanisms of TKI resistance, patients' general situation, patients' wishes, etc. In this case discussion, we present a case of recurrent NSCLC with acquired resistance after gefitinib treatment and discuss the therapeutic strategies under this circumstance. Multidisciplinary experts' opinions played an important role in guiding the treatment for this patient.

\section{Case presentation}

In March 2013, a 59-year-old woman was present at our clinic owing to a pulmonary tumor in the left upper lobe observed during routine chest X-ray examination. After computed tomography-guided biopsy and chest-enhanced computed tomography scan, pulmonary adenocarcinoma was diagnosed. Preoperative brain magnetic resonance imaging and isotope bone scan showed no evidence of distant metastases. Therefore, on April 08, 2013, left upper lobectomy with systemic mediastinal lymph node dissection was performed. The postoperative pathological examination showed a p T1cN2M0 adenocarcinoma with mediastinal lymph node metastases in station 4 and station 11. Subsequently, she received adjuvant chemotherapy with carboplatin $(540 \mathrm{mg})$ and pemetrexed $(800 \mathrm{mg})$ for 4 cycles from May 20 to July 31, 2013.

In January 2017, suspected pulmonary recurrence and brain metastasis were observed during regular postoperative follow-up (Figure 1). To specify the optimal therapeutic strategy, a genetic test of the tumor was performed, and a $L 858 R$ EGFR mutation was discovered, while $A L K$ gene rearrangement and ROS1 gene rearrangement were negative. Therefore, from January 2017, gefitinib (250 mg/ day) was given until December 2018 when progression of the brain metastasis was observed (Figure 2). The brain metastasis in the left temporal lobe enlarged comparing with prior brain magnetic resonance imaging examination. Therefore, seeing as that there was disease progression, second-line treatment was considered.

The patient refused a biopsy of the pulmonary/brain lesions for histological and molecular testing. Thirdgeneration EGFR-TKI was not recommended without the confirmation of the T790M EGFR mutation, while the patient did not wish to pursue chemotherapy. Thus, we treated her with anlotinib $(12 \mathrm{mg} /$ day $)$, an orally 

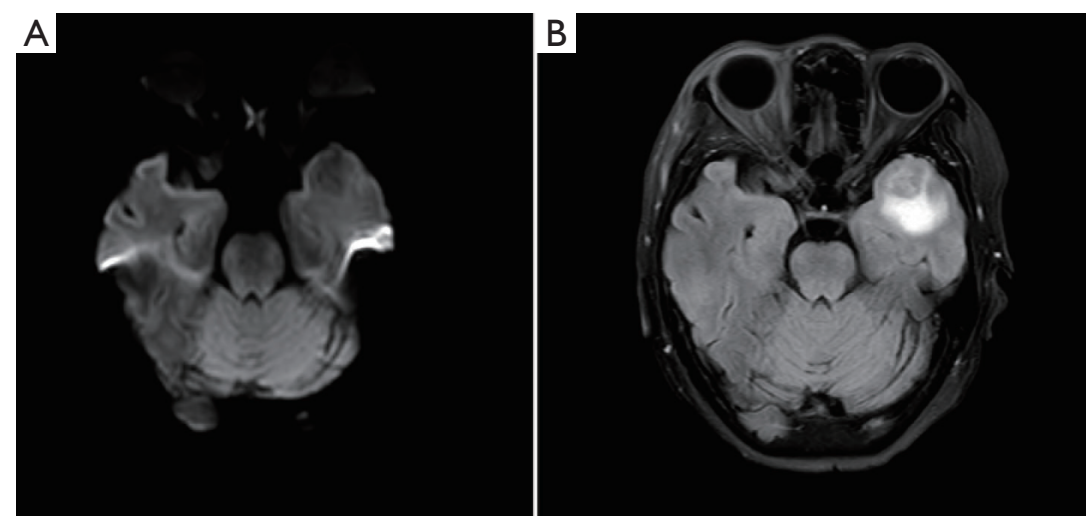

Figure 1 Axial diffusion-weighted (A) and T2W FLAIR (B) brain magnetic resonance imaging on January 23, 2017, showing a new-found brain metastasis in the left temporal lobe.
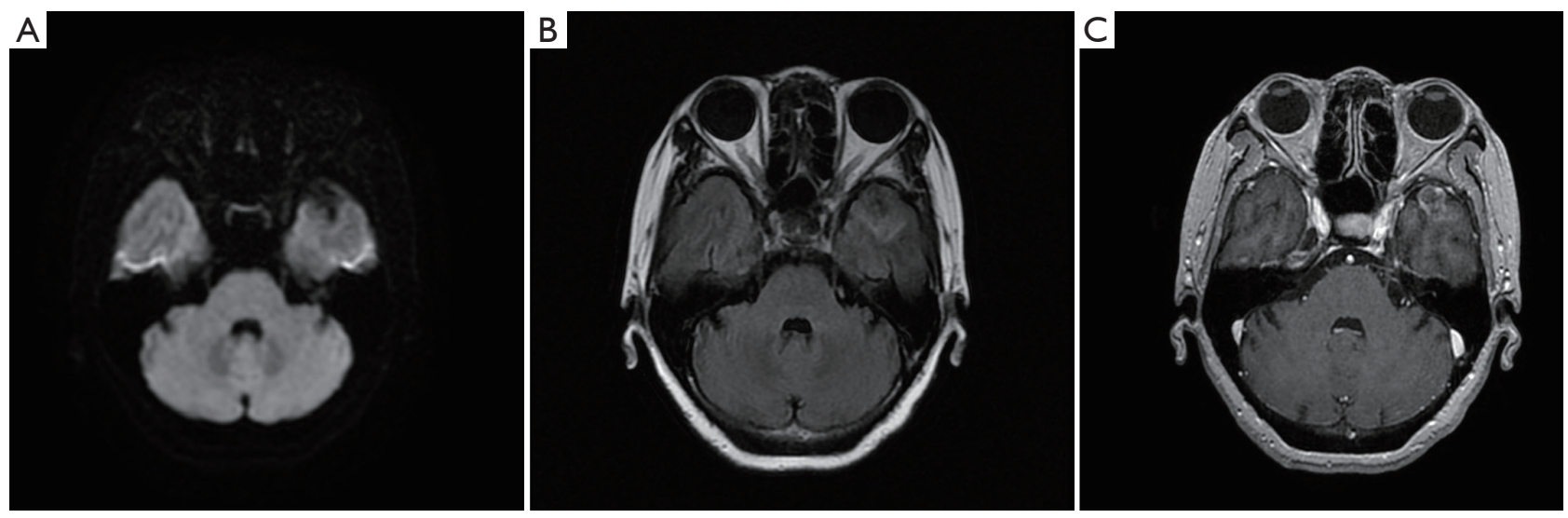

Figure 2 Axial diffusion-weighted (A), T2W FLAIR (B), and contrast-enhanced (C) brain magnetic resonance imaging on December 09, 2018. The metastasis in the left temporal lobe enlarged during gefitinib treatment.

administrated TKI which was recommended as thirdline therapy for advanced NSCLC in China. However, On January 27, 2019, she stopped taking this medication because of severe nausea and headache. Pleasingly, the brain metastasis slightly regressed after about one month of anlotinib treatment (Figure 3).

On February 13, 2019, after the patient consented, a biopsy of a pulmonary lesion was performed, and the T790M mutation was discovered. Therefore, osimertinib was prescribed to the patient. According to the latest chestenhanced computed tomography and brain magnetic resonance examination in August 2019, pulmonary lesions and brain metastasis were evaluated as stable disease based on the Response Evaluation Criteria in Solid Tumors (version 1.1).

\section{Discussion}

The patient was diagnosed with pulmonary adenocarcinoma and underwent left upper lobectomy on April 08, 2013. The pathological examination showed a pT1cN2M0 pulmonary adenocarcinoma and the patient completed 4 cycles of adjuvant platinum-pemetrexed chemotherapy. Four years after surgery, pulmonary recurrence with brain metastasis was discovered. Retrospective molecular testing of the patient's tumor sample confirmed the L858R EGFR mutation. Therefore, gefitinib was given until December 2018 when progression of the brain metastasis was observed. In consideration of the patient's wishes, anlotinib was subsequently used, which was stopped because of severe side 

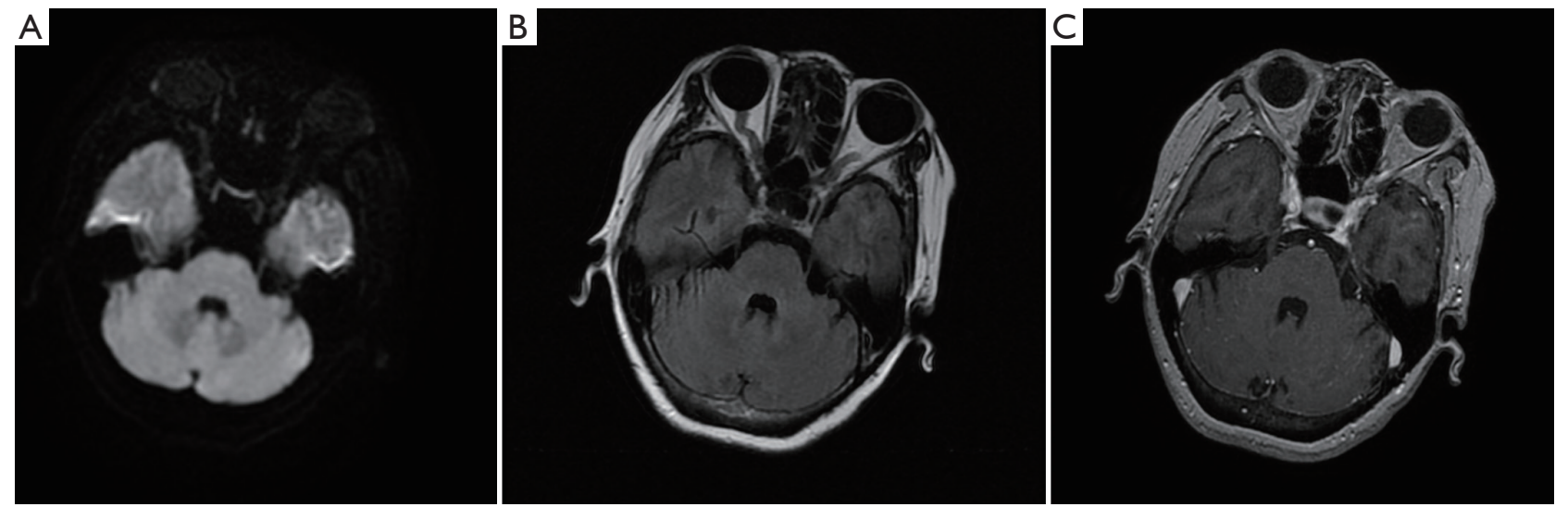

Figure 3 Axial diffusion-weighted (A), T2W FLAIR (B), and contrast-enhanced (C) brain magnetic resonance imaging on February 13, 2019. The metastasis in the left temporal lobe slightly regressed after about one month of anlotinib treatment.

effects a month later. To optimize the following therapeutic strategy for this patient, a multidisciplinary team discussion was conducted.

\section{Department of Thoracic Surgery}

First or second-generation EGFR-TKI shows significant clinical benefit and is widely used as the standard of care for advanced NSCLC harboring an EGFR mutation, with a median progression-free survival (PFS) ranging from 8 to 13 months (1-3). Nevertheless, most patients experienced disease progression because of acquired resistance to EGFRTKIs. Different mechanisms have been elucidated, while the T790M mutation was reported in approximately $60 \%$ of the EGFR-TKI resistance cases $(4,5)$. Other mechanisms include small cell transformation, mesenchymal-epithelial transition factor transformation, etc. In this case, however, the mechanism of gefitinib resistance could not be confirmed due to the patient's unwillingness to receive a second biopsy.

\section{Department of Respiratory Medicine}

Osimertinib, a third-generation EGFR-TKI, is known to have a strong affinity for the T790M mutation in patients with acquired resistance to early-generation EGFR-TKIs. It has been recommended as the optimal therapy for T790M mutation after disease progression with previous EGFR-TKIs. According to the AURA3 study, osimertinib achieved significantly improved PFS compared to platinum and pemetrexed chemotherapy (10.1 vs. 4.4 months) in treating patients with the acquired T790M mutation (6). Accordingly, for patients with the T790M mutation, osimertinib is the optimal choice, while for those without the T790M mutation, chemotherapy is considered as the most widely employed second-line treatment.

However, not every patient previously treated with firstgeneration EGFR-TKI will benefit from osimertinib. According to the prospective observational study conducted by Kanai et al., only $23.70 \%$ of the patients eventually received osimertinib after acquiring resistance to the older-generation EGFR-TKIs (7). In this case, we still strongly recommend a biopsy of the pulmonary or brain lesions in search of acquired T790M mutation, which may significantly improve the patient's prognosis.

For those patients with acquired resistance to EGFR inhibitors who do not harbor T790M mutation, anlotinib, a novel orally administrated receptor tyrosine kinase inhibitor, maybe a choice excluding chemotherapy. According to the phase III clinical trial comparing anlotinib with placebo in patients with advanced NSCLC who progressed after at least 2 lines of prior therapies, anlotinib significantly improved the PFS (5.37 vs. 1.40 months, $\mathrm{P}<0.001)$ and overall survival (OS) (9.63 vs. 6.30 months, $\mathrm{P}<0.001)(8)$. Based on these results, we employed anlotinib in our case. Unfortunately, the treatment was terminated because of apparent side effects, although, the tolerability of anlotinib is generally acceptable (9).

\section{Department of Oncology}

Delayed use of osimertinib may affect the prognosis of 
patients. Prior studies suggested that patients who received osimertinib directly after confirmation of the T790M mutation by rebiopsy had a longer PFS than those with intercalated treatment between rebiopsy and osimertinib (10). However, the patient, in this case, refused the biopsy and received anlotinib as a substitute, which might delay the use of osimertinib and resulted in earlier disease progression. Early biopsy for detecting T790M mutation in progressed NSCLC patients is strongly recommended; liquid biopsy like blood or pleural effusion could also be used as an alternative in the clinic.

An emerging question is whether the early use of osimertinib provides a superior outcome in the first-line setting. According to the recent phase 3 FLAURA trial, osimertinib was demonstrated as a viable first line therapy in NSCLC with EGFR mutation (11). Moreover, osimertinib was considered to be more efficacious than standard EGFRTKIs for patients with central nervous system progression (12). The initial use of osimertinib could also be a promising option, especially for patients with a high risk of brain metastases. Recently, it has been recommended as the preferred first-line therapy for advanced NSCLC.

\section{Several questions should be further discussed}

Question 1: If the patient still insists on not receiving biopsy and chemotherapy, what's the substitution of treatment?

\section{Expert opinion 1: Dr. Surein Arulananda}

Central nervous system (CNS) disease progression on EGFR-TKIs is a fairly common situation that we face in the clinic. CNS disease progression could be a consequence of pharmacokinetic failure of the 1st generation EGFR-TKIs rather than resistance mutations. In the first instance, a plasma or cerebrospinal fluid based liquid biopsy to look for the T790M mutation is suggested. If the T790M mutation is present, I would start the patient on osimertinib, which we know has highly potent CNS activity. If there is no T790M mutation, this could be a false negative and it would be reasonable to switch the patient's treatment to pulsed EGFR-TKI dosing (i.e., 1,500 mg/week of erlotinib) or even to osimertinib $(80 \mathrm{mg} /$ day) to attempt an increase in cerebrospinal fluid penetrance. However, another good option is that if the patient has oligometastatic disease progression in the brain, I would refer the patient for stereotactic radiosurgery. The local control rates are high, and I would continue the 1st generation EGFR-TKI but watch the patient closely for further disease progression.

\section{Expert opinion 2: Dr. Sang-Won Um}

The patient had CNS failure in the absence of systemic failure at the time of the progression of brain metastasis after gefitinib treatment. Therefore, a possibility of CNS sanctuary progressive disease should be also considered for this patient (13). CNS sanctuary progressive disease is due to poor penetrance of 1st-generation EGFR-TKI (gefitinib) into the brain rather than emergence of resistant tumor clones (13). Stereotactic radiosurgery for the brain metastasis while continuing gefitinib therapy could be a therapeutic option if the patient still refuses to receive biopsy/chemotherapy and plasma EGFR T790M testing is negative.

Question 2: For this patient, will you choose osimertinib as first line therapy instead of first or second-generation EGFR-TKIs?

\section{Expert opinion 1: Dr. Surein Arulananda}

The FLAURA study met both its primary end-point of PFS and secondary end-point of OS. Osimertinib also demonstrated improved CNS response rates and prolonged time to CNS progression compared to 1 st generation EGFR-TKIs. Moreover, osimertinib had an improved toxicity profile over the 1st generation TKIs. Having said that, in the Forrest-plot subgroup analyses, the hazard ratio for Asian patients and $L 858 R$ mutations (both of which apply to our patient) appeared to be equivocal for osimertinib or 1st generation EGFR TKIs. I would still consider this to be exploratory and am at least reassured that there was no worsening in outcomes. Therefore, for all these reasons, I would choose osimertinib as $1^{\text {st }}$ line therapy in this patient.

\section{Expert opinion 2: Dr. Sang-Won Um}

Yes. The FLAURA trial demonstrates a progression-free survival benefit in the whole population as well as in the patients with CNS metastases, which supports osimertinib as first line therapy in patients with EGFR mutated advanced non-small cell lung cancer with brain metastasis.

\section{Question 3: There exist several different first or} second-generation EGFR-TKIs, including gefitinib, afatinib, erlotinib, etc., which drug is preferred as first line therapy for NSCLC patients? Why? Expert opinion 1: Dr. Surein Arulananda

As addressed in question 2, osimertinib is preferred as firstline therapy for EGFR mutant NSCLC patients. However, if this is not an option, then the choice of drug would be patient dependent. For example, afatinib is preferred in 
patients with de novo brain metastases as it appears to have the best CNS response compared to erlotinib and gefitinib. For patients who are older and frailer due to co-morbidities, gefitinib is preferred as it is usually well tolerated. For non-Asian patients, erlotinib is generally used as per the EURTAC study.

\section{Expert opinion 2: Dr. Sang-Won Um}

I will choose second-generation EGFR-TKI (afatinib) among first or second-generation EGFR-TKIs. Previous head-to-head comparison studies [ARCHER (dacomitinib $v s$. gefitinib) and Lux Lung 7 (afatinib vs. gefitinib)] suggested the superiority of second-generation EGFRTKIs over the first-generation EGFR-TKIs in terms of progression-free survival or OS at the cost of slightly more toxicity $(14,15)$.

\section{Conclusions}

Acquired resistance to first or second-generation EGFRTKIs constitutes a significant clinical problem for advanced NSCLC treatment. Management of NSCLC harboring $E G F R$ mutations should be thoroughly considered, especially in difficult circumstances.

With the help of a multidisciplinary team discussion, we summarized and shared the status and research progress of therapeutic strategies for patients with acquired EGFRTKI resistance. The multidisciplinary discussion also plays an important role in promoting personalized medicine.

\section{Acknowledgments}

Funding: This work was supported by the National Natural Science Foundation of China (81871882), Shanghai Municipal Education Commission-Gaofeng Clinical Medicine Grant Support (20172005) and Shanghai Municipal Commission of Health and Family Planning Outstanding Academic Leaders Training Program (2017BR055).

\section{Footnote}

Conflicts of Interest: HL serves as the unpaid editorial board member of Fournal of Thoracic Disease from Jul 2018 to Jun 2020. S Arulananda declares personal fees from Merck-Sharpe Dohme, Astra Zeneca, Boehringer-Ingelheim and Roche. The other authors have no conflicts of interest to declare.

Ethical Statement: The authors are accountable for all aspects of the work in ensuring that questions related to the accuracy or integrity of any part of the work are appropriately investigated and resolved. Written informed consent was obtained from the patient for publication of this case report and any accompanying images.

Open Access Statement: This is an Open Access article distributed in accordance with the Creative Commons Attribution-NonCommercial-NoDerivs 4.0 International License (CC BY-NC-ND 4.0), which permits the noncommercial replication and distribution of the article with the strict proviso that no changes or edits are made and the original work is properly cited (including links to both the formal publication through the relevant DOI and the license). See: https://creativecommons.org/licenses/by-nc-nd/4.0/.

\section{References}

1. Mok TS, Wu YL, Thongprasert S, et al. Gefitinib or carboplatin-paclitaxel in pulmonary adenocarcinoma. $\mathrm{N}$ Engl J Med 2009;361:947-57.

2. Ma Y, Xin S, Lin Q, et al. The analysis of pharmacokinetic and pharmacogenomic impact on gefitinib efficacy in advanced non-small cell lung cancer patients: results from a prospective cohort study. Ann Transl Med 2019;7:806.

3. Yoshida K, Kanda S, Shiraishi H, et al. Difference in central nerve system metastasis during gefitinib or erlotinib therapy in patients with EGFR-mutated nonsmall cell lung cancer: a retrospective study. J Thorac Dis 2019;11:1347-54.

4. Yu HA, Arcila ME, Rekhtman N, et al. Analysis of tumor specimens at the time of acquired resistance to EGFR-TKI therapy in 155 patients with EGFR-mutant lung cancers. Clin Cancer Res 2013;19:2240-7.

5. Lettig L, Sahnane N, Pepe F, et al. EGFR T790M detection rate in lung adenocarcinomas at baseline using droplet digital PCR and validation by ultra-deep next generation sequencing. Transl Lung Cancer Res 2019;8:584-92.

6. Mok TS, Wu YL, Ahn MJ, et al. Osimertinib or PlatinumPemetrexed in EGFR T790M-Positive Lung Cancer. N Engl J Med 2017;376:629-40.

7. Kanai K, Yamamoto N, Nogami N, et al. 141PD A prospective study of molecular testing status in the EGFR mutation positive NSCLC patients with disease progression during EGFR TKI treatment (REMEDY study). J Thorac Oncol 2018;13:S82-S3.

8. Han B, Kai L, Wang Q, et al. Effect of Anlotinib as a 
Third-Line or Further Treatment on Overall Survival of Patients With Advanced Non-Small Cell Lung Cancer: The ALTER 0303 Phase 3 Randomized Clinical Trial. JAMA Oncol 2018;4:1569-75.

9. Shen G, Zheng F, Ren D, et al. Anlotinib: a novel multitargeting tyrosine kinase inhibitor in clinical development. J Hematol Oncol 2018;11:120.

10. Tseng JS, Yang TY, Chen KC, et al. Intercalated Treatment Following Rebiopsy Is Associated with a Shorter Progression-Free Survival of Osimertinib Treatment. Cancer Res Treat 2018;50:1164-74.

11. Soria JC, Ohe Y, Vansteenkiste J, et al. Osimertinib in Untreated EGFR-Mutated Advanced Non-Small-Cell Lung Cancer. N Engl J Med 2018;378:113-25.

12. Gourd E. CNS efficacy of osimertinib in EGFR-mutated

Cite this article as: Zheng $\mathrm{Y}$, Zhou M, Arulananda S, Um SW, Li H. Management of non-small cell lung cancer with resistance to epidermal growth factor receptor tyrosine kinase inhibitor: case discussion. J Thorac Dis 2020;12(3):159-164. doi: $10.21037 /$ jtd.2020.01.65 advanced NSCLC. Lancet Oncol 2018;19:e516.

13. Gandara DR, Li T, Lara PN, et al. Acquired resistance to targeted therapies against oncogene-driven non-small-cell lung cancer: approach to subtyping progressive disease and clinical implications. Clin Lung Cancer 2014;15:1-6.

14. Mok TS, Cheng Y, Zhou X, et al. Improvement in Overall Survival in a Randomized Study That Compared Dacomitinib With Gefitinib in Patients With Advanced Non-Small-Cell Lung Cancer and EGFR-Activating Mutations. J Clin Oncol 2018;36:2244-50.

15. Park K, Tan EH, O'Byrne K, et al. Afatinib versus gefitinib as first-line treatment of patients with EGFR mutationpositive non-small-cell lung cancer (LUX-Lung 7): a phase 2B, open-label, randomised controlled trial. Lancet Oncol 2016;17:577-89. 